\title{
Commentary
}

\section{Key elements in a strategic approach to capacity building in the biosafety of genetically modified organisms*}

\author{
Marianela ARAYA-QUeSADA, Giuliano DegrassI, Decio RIPANDelLI and Wendy CrAiG** \\ Biosafety Unit, International Centre for Genetic Engineering and Biotechnology (ICGEB), Padriciano 99, TS 34149, Italy
}

In recent times, it has become imperative for countries to define and implement policy in biosafety due to the widespread adoption of genetically modified crops. As such, countries wishing to utilise transgenic technologies in the development of advanced agricultural products must have regulations in place coupled with trained personnel in national competent authorities able to contribute effectively to the decision-making process. Capacity building initiatives play an important role in supporting such individuals, institutions and governmental authorities by providing training and/or physical structures/equipment and technical assistance. There are many types of capacity building activities; however not all have the same relevance in different regions of the world. For capacity building to be effective, a strategic approach incorporating a variety of forms and disciplines is desired. This commentary discusses the importance of factors such as: the targeting of support to relevant beneficiary(ies); the identification of specific needs and the incorporation of socio-economic conditions when elaborating effective strategies designed to help building capacity. Moreover, the importance of interaction and collaboration amongst the various capacity builders is also discussed such that unnecessary duplication of efforts and best use of available human and economic resources results.

Keywords: biosafety needs / capacity building / genetically modified organisms (GMOs) / decision-making

\section{INTRODUCTION}

The concept of biosafety encompasses a number of different measures, precautions, policies and actions to be taken for minimising potential risks that biotechnology and its various applications may pose to the environment and human health (FAO, 2009b). In recent years, it has become imperative for countries to act promptly to define and implement policy in biosafety, primarily due to the widespread adoption of genetically modified (GM) crops (James, 2009), the ratification and implementation of the Cartagena Protocol on Biosafety (CPB) (Secretariat of the Convention on Biological Diversity, 2000, 2009), together with all the issues related to trade activities involving genetically modified organisms (GMOs) (Johnston et al., 2008).

It is generally recognised that biosafety considerations are an integral part of the development of agricultural biotechnology products, especially GM crops,

\footnotetext{
* Disclaimer: Statements and opinions expressed in this manuscript are those of the authors and do not necessarily correspond to ICGEB policy or opinion.

** Corresponding author: craig@icgeb.org
}

ultimately destined for the marketplace. No GMO or derived product will gain market authorisation without a full evaluation of the potential risks (and in some jurisdictions, also benefits) arising from their deployment. As such, it is evident that, for those countries wishing to utilise the technology or its products, there must be regulations in place, and that members of national competent authorities, their scientific advisers, product developers, decision-makers and stakeholders in general are able to contribute effectively to the regulatory risk assessment, risk management and decision making processes.

This underlines the increasing importance that countries have the necessary "capacity" to manage the challenges related to the adoption of biotechnological products, or else, must elaborate strategies to develop such capacity with the aim of creating a bedrock of relevant knowledge and expertise. Therefore, the concept of biosafety capacity building has emerged to provide support in achieving national and/or international biosafety proficiency.

Acknowledging that capacity building means different things to different people, and that it can be applied to many disciplines, for the purposes of this paper it is 
meant as "the process by which individuals, organizations, institutions and societies develop abilities (individually and collectively) to perform functions, solve problems and set and achieve objectives" (ILO, 2005). Moreover, capacity building often implies the provision of technical assistance, training, and specialist inputs, in addition to financial resources (OECD, 2002). These latter provisions were already recognised as key to the effective implementation of the Cartagena Protocol on Biosafety (CPB), the first binding international agreement dealing with biosafety. Indeed, an entire article (Article 22) of the CPB is dedicated to capacity building and requires Parties to cooperate in the development and strengthening of biosafety capacities: "In order to be able to implement their obligations, Parties need appropriate institutional mechanisms and infrastructure, well-trained human resources, adequate funding as well as easy access to relevant information" (Secretariat of the Convention on Biological Diversity, 2000).

Creating capacity in biosafety represents a challenge for any institution or individual involved ("capacity builders"). This is principally due to the fact that expertise in a number of scientific, technical and legal disciplines is needed (Craig et al., 2008, 2010), and it is highly unlikely that one capacity builder alone can provide all the necessary support. However, capacity building can be "casespecific" and can also take different forms depending on the objectives of the initiative, the expertise of the team members and the characteristics of the target (beneficiary). The different forms can for instance focus on supporting individuals, or helping institutions or governmental authorities, whilst other forms of support may involve providing training and/or physical structures/equipment. Nevertheless, for capacity building to be effective and efficient, a strategic approach incorporating a variety of forms and areas is desired. Moreover, there are many factors that play an important role in the success or failure of a biosafety capacity building activity (economic resources, timeframes, technical expertises, defined needs), some of them related to the stakeholders involved, especially considering that "stakeholder" is taken to mean a person or organisation with a legitimate or vested interest (a "stake") in a project or entity.

This commentary will discuss some of the key elements that form part of a strategic approach for developing capacity building in biosafety, taking advantage of the information and experiences generated from the current ICGEB biosafety capacity building initiative in sub-Saharan Africa (SSA), and complemented by the outcome of a recent stakeholder consultation in Latin America and the Caribbean (LAC) (ICGEB, unpublished data), with the aim of highlighting the importance of planning and coordination during the development of capacity building activities, alongside cooperation and interaction with different capacity building initiatives.

\section{STRATEGIC APPROACH}

\section{Defining the target: Capacity building for developing countries - an example}

It is clear that biosafety capacity building is greatly needed around the world, and that even advanced biosafety regulatory frameworks in developed countries require continual capacity building in order to keep up with the latest developments of the technology and the turnover in staff. Nevertheless, in the majority of cases, greater support is required by developing countries, especially regarding technical training and the development of national biosafety systems, largely due to the lack of local resource personnel with sufficient experience in the numerous scientific and technical fields that pertain to biosafety (ICGEB, unpublished data).

The regulation of GMOs is but one of a multitude of institutional areas that the majority of developing countries are struggling to deal with, and for which an "ad hoc" strategy has been adopted in many cases. Territories with this approach include most countries of Africa, Central Asia, Oceania and the Caribbean, as well as some parts of Latin America (Johnston et al., 2008; ICGEB, unpublished data). A contributing factor is that biotechnologists are generally first employed in research and development rather than in regulatory services, therefore minimising the opportunities of new personnel to actually focus in obtaining relevant biosafety knowledge and expertise (Louwaars et al., 2002). At the same time, few, if any, permanent positions are open for biosafety experts in the local competent authorities of many developing countries. In the majority of cases the personnel involved are either not sufficiently financially remunerated or must perform the role on an ad hoc basis. In addition, many of the members of competent authorities have received little training in biosafety, which therefore limits their abilities and renders the decision-making process more difficult.

Considering these limitations on possible areas to support, the definition of the focus (target) of a biosafety capacity building initiative becomes an extremely important step for the whole project, and of course must be strictly related to the ultimate final goal of the initiative. Selecting inappropriate beneficiaries is likely to result in a minimal or partial achievement of project objectives and may even be a waste of time, money and human resources. For instance, considering that the institutions key to biosafety regulation may be present in name only, it may not always be obvious as to which 
individuals training should be addressed. In such a scenario, a detailed and careful identification of candidates is advisable, with more upstream energies devoted towards gathering information concerning the role of the various actors and institutions involved in the process before any training or support is offered. Furthermore, the diversity of public opinions and national policies, as well as the different possibilities and capabilities of countries and regions to assess the implications (positive or negative) of new biotechnology products based on their own experience, are key elements to be considered when identifying the target of a capacity building strategy. Moreover, differences within the possible targets must be taken into account and aligned with the capacities and expertise of the capacity builder. In other words, three major points should be considered for the selection of the target: 1) What is needed? Is it technical training, infrastructure, regulatory system, legislative instruments, etc.?; 2) Who needs it? Countries, institutions, individuals, etc.?; and 3) What can the capacity builder do to fulfil the need(s)?

\section{Identifying and interpreting needs}

Identification of the main needs of the target (region, country, institution, individuals, etc.) is important in designing a capacity building strategy. Capacity builders should be able to adapt their strategy(ies) to target beneficiary needs and realistic local conditions, and not the opposite. Many objectives can be defined, but the overall goal should be to make a noticeable improvement based upon a comparison with the previous prevailing conditions. Frequently, targeting specific gaps permits a more effective and efficient use of available economic and human resources.

When identifying needs, a useful approach is to perform a stakeholder consultation with the aim of gaining sufficient knowledge of the local situation, e.g. strengths and weakness in the area of interest, different types of stakeholders, local policies, socio-economic aspects, etc. The identification of specific requirements or needs should form the first step in any biosafety capacity building initiative. Experience has demonstrated that a variety of key stakeholders should be consulted, including researchers, scientists, governmental officers, regulators, politicians, farmers, and consumers. Consulting with such a heterogeneous group, and combining the outcomes with feedback from any biosafety capacity builders currently operating in the region, ensures a comprehensive coverage, even if not all the identified needs can be addressed in the proposed initiative. In addition, as biosafety is open to many interpretations, such a consultation should ensure that the outcome fairly reflects the local situation, especially when the various stakeholders may have slightly differing perspectives and opinions, eventually enabling a more complete and specific action plan to be elaborated. For example, one group of stakeholders may identify the need for developing administrative guidelines in general, whereas another group may be more specific and identify the need for guidelines for handling notifications or applications for import/export of GMOs only. An adjunct is that it is not just a matter of consulting a large and varied number of stakeholders, but there must also be reliable sources of information such that their feedback reflects the reality of the situation. In certain cases, consulting with only a few well-informed stakeholders may result in the development of a more successful strategy than relying on the outcome from a broader, but less-informed consultation.

Notably, although developing countries may share a similar set of biosafety capacity building needs, they may also exhibit regional variation in the extent of these needs. For example, both LAC and SSA have an immense need of support in order to achieve fully operational biosafety systems, however, when both regions are analysed, clear differences within the present regulatory expertise can be perceived, which will ultimately impact the type of support provided. For example, there are more countries in LAC region (as compared to SSA) with GM products: at the lab/greenhouse stage ( 9 versus 6 ); confined release (10 versus 6$)$; and commercialised GM products (8 versus 2) (ICGEB, unpublished data). At the same time, identified training needs in SSA were of a more generic nature (risk assessment strategies, understanding the biology of the main crops in the region, or risk analysis, amongst others) than the more defined needs of the LAC region (consequences of gene flow, possible effects on non-target organisms, increase in allergenicity potential, possible toxicity of GM products and managing pest resistance) (ICGEB, unpublished data). So, whilst there is an evident need for biosafety capacity in both regions, the type required to fulfil each need may or may not be the same. Moreover, frequently when a stakeholder consultation is performed, the particular aspects of biosafety where training or support is required are not always clearly stated. For example, "Risk assessment and other scientific and technical expertise" was identified as one of the "key elements requiring concrete action" by Parties to the CPB (Secretariat of the Convention on Biological Diversity, 2004). However the precise nature of this element was not further elaborated, nor was it specified whether it was with regard to importation, for the purposes of confined field trials, open cultivation, or direct use as food, feed or processing, or to all these purposes. This is particularly salient, as the risk assessment paradigm is different for each purpose. Therefore, it is the responsibility of the capacity builder(s) to ensure that they fully understand the extent of the need(s) in question. Another example is that the most common generic need, 
as identified by at least 50 developing countries Parties was "institutional building" (e.g. regulatory and administrative capacity, infrastructure, funding and mechanisms for follow-up), followed by "human resource development and training" (Secretariat of the Convention on Biological Diversity, 2005).

\section{Selecting the best approach: Different types of capacity building}

Building capacity in such a diverse arena as biosafety is complicated by the fact that there is no a single best approach for specific stakeholders who range from local governments, to communities and NGOs, or from central governments to the private commercial sector. Therefore a case-by-case approach should be adopted whenever developing programmes (McLean et al., 2002). Additionally, capacity building needs are constantly evolving and any programme under development must take into consideration the current local situation, the organisation/individual to which it is addressed, and the sustainability of the project.

Many different types of capacity building activities exist, including: 1) at the individual level i.e. human resource development, in the form of imparting knowledge and developing skills to enable them to perform effectively; 2) at the institutional level i.e. organisational development, such as the creation of management structures and processes within the public, private and community sectors, and developing the ability to adapt to change; and 3) at the systemic level i.e. institutional and legal framework development, emphasising the overall policy framework in which individuals and organisations operate and interact with the external environment, as well as the formal and informal relationships of institutions. It may also involve the creation of legal and regulatory instruments to enable governments, institutions, and agencies to improve their effectiveness (GDRC, 2009). Each project must select the most pertinent approach for each specific situation.

As biosafety needs differ around the world, proven solutions are important foundations (especially at the local level) upon which to build further capacity. It has been proposed that capacity builders develop more effective methods for promoting local projects and explore innovative locally-tailored programmes in order to first address specific needs before turning their attentions to global requirements (FAO Biotechnology Forum [Worku], 2009; Johnston et al., 2008), arguing that only when individual competences have been achieved will global requirements be more effectively addressed.

At times, a simple approach covering key aspects for addressing gaps can be far more effective than one that is more elaborate but less specific. For example a capacity builder with experience in providing technical biosafety training may focus efforts in solving educational needs, and leave efforts towards supporting the development of other aspects of the biosafety regulatory system to another capacity builder with the relevant expertise in regulatory processes. Alternatively, different approaches can made concomitantly to address the same gaps. For instance, when a specific biosafety training need has been identified, then the next step is to define the most effective approach for its provision. This may be through shortterm local courses, long-term training activities, training abroad and/or through distance learning programmes. All of these are possible options that can be used to attend to the same need, however the effectiveness of each will rely on the conditions restricting their uptake by the target beneficiary, e.g. socio-economic limitations, no authorised extended absence from work, inability to travel, or even the amount of hours that can be dedicated to training per day (One World, 2010; ICGEB, unpublished data).

Once needs have been identified, the developed strategic approach should take into account whether the beneficiary (country, region, institution, etc.) is actually able to derive benefits from the capacity building efforts. For instance, regional initiatives to establish centres of excellence, laboratory infrastructure, and regional training programmes could ultimately prove useless if a regional policy is not in place to ensure "ownership" and acknowledgement of the responsibilities governing the use of such facilities, expertise, etc. (Secretariat of the Convention on Biological Diversity, 2005). Moreover, the provision of sufficient economic resources is paramount in ensuring the success and sustainability of the project. For example, funds devoted towards the construction of a GMO detection laboratory or biosafety research facility are wasted if there is no clear mechanism by which operational resources (e.g. human, logistic and consumable) are maintained beyond the project lifetime.

\section{Combining efforts results in a more effective strategy}

When considering implementing a biosafety capacity building initiative, it is always extremely useful to consider the legacies, both short-lived and enduring, of previous initiatives in order to build on previous good work and not repeat the failings of less effective work. There are many institutions currently engaging in biosafety capacity building, such as: the Food and Agriculture Organization of the United Nations (FAO); the International Centre for Genetic Engineering and Biotechnology (ICGEB); the Program for Biosafety Systems (PBS), managed by the International Food Policy Research Institute (IFPRI); the United Nations Environment 
Key elements in a strategic approach to capacity building in the biosafety of genetically modified organisms

Programme (UNEP); and the United Nations Industrial Development Organization (UNIDO), amongst many others. These are adding to the work of previous initiatives, sometimes by the same institution, that was carried out worldwide in the last 15-20 years (FAO, 2009b; Johnston et al., 2008). Therefore undertaking a detailed study of their outcomes is clearly advisable to avoid both "a reinvention of the wheel" and duplicating efforts, thus making better use of limited time and available resources. Incorporating and further expanding the strategic approach during the planning phase of a capacity building initiative, although requiring a significant amount of time and effort, is hugely rewarding, especially as it should result in a refinement of project goals and methods of how to achieve them, being based on more informed decisions and more effective use of economic resources.

With so many capacity building initiatives underway, it is hardly surprising to find that a degree of overlap has occurred, either in terms of the activities undertaken or countries where the initiatives have been implemented. For example, there are cases of several projects being implemented by different organisations each providing assistance for the development or revision of the same national biosafety framework. Further, multiple workshops have offered training on the same issues (i.e. risk assessment) to the same target groups, e.g. National Biosafety Committee members (Secretariat of the Convention on Biological Diversity, 2005). It is obvious that no single institution is able to address the present wide range of biosafety needs. Therefore, cooperation amongst capacity builders is a logical strategy to pursue that will allow the various and different expertises and knowledge to interact and complement one another, if not ultimately to be pooled, in attaining common objectives. This will also allow capacity builders to focus more energies within their field(s) of expertise, and facilitate positive interventions that may stretch beyond individual competencies. Furthermore, actions can then be prioritised in order to achieve a broader, deeper coverage and make best use of the economic and human resources dedicated to solve biosafety needs (Johnston et al., 2008). It is acknowledged however, that obtaining desirable synergy amongst multiple capacity building organisations will most likely be constrained to those activities that meet mutual operational and philosophical objectives.

At the same time, combining efforts should not be perceived only as a policy or attitude for capacity builders, but it can also become a strategy for beneficiaries (e.g. neighbouring countries) to optimise the use of available resources or expertises when there are common aims. Thus, while certain issues remain unique for each country (i.e. formulating national biosafety policies and legislation), common needs (e.g. well-established capacities to develop a regulatory framework) offer the opportunity for regional collaboration and harmonisation for mutual benefit (FAO, 2009b). Examples of possible cooperation include the establishment of: centres of excellence, regional information centres, laboratory infrastructure, LMO testing and monitoring facilities, model biosafety laws or guidelines, and regional training programmes. Furthermore, regional cooperation/networks support the sharing of information and experience amongst the involved countries, thereby more effectively utilising funds in specific activities, harmonising policies, regulation, and trade practices, etc.

\section{Socio-economic considerations as part of capacity building strategies}

The consideration of socio-economic differences amongst beneficiaries plays an important role in the planning and implementation of capacity building activities. Differences in common languages, the level of current scientific and technical knowledge and expertise, the availability of operational facilities and infrastructure, the political situation, available economic resources and even cultural beliefs and practices become key elements when defining the scope, methods of implementation, and likely impact of a project, to the extent that they could play a determinant role in the eventual success or failure of the project. For instance, any language barrier will obviously add a large degree of difficulty to offered training, thus translation into the local language(s) is highly recommended (FAO, 2009b). Complementing this approach is the provision of training materials in different languages and the use of multilingual resource personnel. Notably, it is interesting that relatively few projects have been reported to be addressing socio-economic considerations (Secretariat of the Convention on Biological Diversity, 2005). Further, should beneficiaries be required to be absent from work for an extended period in order to take up training offerings (e.g. study abroad, internships, etc.), it should first be determined that any necessary permission and/or remunerations are obtainable. Many other local conditions can significantly impact project outcomes. For the purposes of this commentary, a search of the Biosafety Clearing House (BCH) and internet resources of capacity builders currently operating in this field has indicated that the situation remains relatively unchanged.

\section{Assessment of the project impacts}

The assessment of past and present capacity building efforts is of paramount importance for developing future strategies. It is in the capacity builder's best interests to carry out some form of impact assessment, even if it might only result in subjective opinions. Previous 
methods have been the use of surveys, undertaking personal interviews with key stakeholders, and even formal examinations in cases where they have been possible. Lately, though, there has been a push towards the incorporation of indicators to monitor project implementation (Secretariat of the Convention on Biological Diversity, 2010). However these are difficult entities to identify in the world of biosafety where any progress is dependent upon the combined outcomes of multiple, interdependant factors. For instance, an assessment of any long-term impacts may involve a comparison of the "before and after" profiles of the beneficiary (country, institution or individual), and combining this with indicators such as the proclamation of specific biosafety laws or acts, the publication of specific documents and guidelines, the implementation of monitoring systems, the construction of physical facilities, the training of local experts, etc. Whenever such an impact assessment is to be undertaken, the selected methodology should be as objective as local circumstances allow.

Any assessment should also consider the degree to which the impact is sustainable beyond the project lifetime (Secretariat of the Convention on Biological Diversity, 2009), as this will depend as much on the processes of capacity building as on the output of the project (UNEP-GEF Biosafety Unit, 2005). Some approaches shown to help ensuring sustainability include: the strategic engagement of volunteers; training key stakeholders in management activities; the use of online peer group networking, building alliances; and the improvement of fund-raising and donor relationship skills (One World, 2010). For instance, many countries have had several short-term biosafety capacity-building initiatives that have never been expanded upon or followed up to ensure a successful impact (Secretariat of the Convention on Biological Diversity, 2009). Adding to the problem is that sustainability is constantly being challenged by the frequent turnover of personnel in regulatory agencies or in other institutions that are part of the biosafety system. Further, many government institutions have difficulty in obtaining sufficient biosafety budgets, especially when the related political priority declines. Therefore, strategies devoted to creating in-house knowledge (e.g. training of trainers) stand a greater chance of maintaining longterm continuity (FAO, 2009b).

Finally, it is advisable to carry out any impact assessment activities during the lifetime of the initiative so that there is time to respond and/or adapt to any useful feedback generated. Should this approach be impossible or not carried out during project implementation, it should at least be done at the end of each capacity building activity. Dissemination of such information is highly encouraged in order to improve the likelihood of success of future activities.

\section{CONCLUSIONS}

Many factors play an important role in biotechnology development and acquisition, transforming countries either into advanced biotech developers (those producing new biotechnology products and using advanced technologies or procedures) or into more modest science users (mainly technology importers) (FAO Biotechnology Forum [Oyewole], 2009; James, 2009). Any likely application of the technology is highly dependant upon: 1) the country's disposition towards possible adoption or implementation; 2) an assessment of the benefits and risks; 3 ) the level of technical mastery; and 4) the provision of adequate non-human resources. The need for biosafety regulatory systems is becoming more obvious for countries to deal with new biotechnology applications and products. Thus support in such broad disciplines should be carefully planned and strategically developed, aiming to optimise the use of the available resources.

There are many types of capacity building activities; however, not all of them have the same relevance for different parts of the world. When selecting the best strategic approach, the capacity builders should be aware of aspects such as: their own area of competence, the kind of beneficiary (individual, institution, private, governmental, etc.) and the requirements of the target audience. Recognition of strengths and weakness both from the capacity builder and from the target, will always result in better accomplishment of the initial project objective and in more real and sustainable efforts. At the same time, cooperation, strategic alliances and partnerships could be a better strategy when the needs are such that not a single initiative is sufficient. Moreover, networking amongst capacity builders generates a better perception and sense of the target's needs whilst being aware of overlapping inputs and hence avoiding duplication of efforts.

Experience has proved that socio-economic considerations plays an important role in the success of any capacity building initiative, therefore, a clear conception of the characteristics, behaviours, and customs of the beneficiaries is of primary importance to ensure the success and sustainability of the project impacts. It is true that there is a tendency to generalise when identifying and addressing needs; such as in the case of developing countries, by presuming that they do not have expertise in applying technologies, assessing the risk of GMOs or developing regulatory frameworks. However, developing countries and any other target, should not be treated as a homogenous block, given the diverse range of economic development, socio-cultural aspects and their specific biotechnology and biosafety needs (FAO Biotechnology Forum [Sharry], 2009). Likewise, an integral part of any capacity building project should be the flexibility, to ensure response to the beneficiaries' changing needs as they evolve over the lifetime of the project. It should be borne 
Key elements in a strategic approach to capacity building in the biosafety of genetically modified organisms

in mind, that capacity builders should adapt to the beneficiary's needs and not expect the beneficiary to adapt to its aptitudes.

Received August 6, 2010; accepted August 20, 2010.

\section{REFERENCES}

Craig W, Tepfer M, Degrassi G, Ripandelli D (2008) An overview of general features of risk assessments of genetically modified crops. Euphytica 164: 853-880

Craig W, Araya-Quesada M, Lewandowski R, Degrassi G, Ripandelli D (2010) An overview of online databases and web-based resources related to the biosafety of genetically modified crops. Collection of Biosafety Reviews (to appear)

FAO Biotechnology Forum (2009a) Learning from the past: Successes and failures with agricultural biotechnologies in developing countries over the last 20 years. Conference 16, FAO Biotechnology Forum [commentator], Food and Agriculture Organization of the United Nations (FAO), Rome, Italy. http://www.fao.org/biotech/conf16.htm

FAO (2009b) Building Biosafety capacity: FAO's experience and outlook. An overview of the experience gained from FAO capacity building projects in agricultural biotechnology and biosafety. Food and Agriculture Organization of the United Nations (FAO), Rome, Italy. http://www.fao.org/docrep/012/ i1033e/i1033e00.htm

GDRC (2009) About the GDRC: Capacity Building Policy. The Global Development Research Center. http://www.gdrc.org/ about/cb-policy.html

ILO (2005) Thesaurus. Department of Communication and Public Information, Bureau of Library and Information Services, International Labour Organization (ILO), Geneva, Switzerland. http://www.ilo.org//thesaurus/defaulten.asp

James C (2009) Global Status of Commercialized Biotech/GM Crops: 2009. International Service for the acquisition of Agri-biotech Applications (ISAAA) Brief 41, Ithaca, NY, USA. http://www.isaaa.org/resources/publications/briefs/41/ executivesummary/default.asp

Johnston S, Monagle C, Green J, Mackenzie R (2008) Internationally-funded training in biotechnology and biosafety: Is it bridging the biotech divide? United Nations University, Institute of Advanced Studies (UNU-IAS), Yokohama, Japan. http://www.ias.unu.edu/sub_page.aspx? cat $I D=111 \&$ dd $1 \mathrm{ID}=673$

Louwaars N, Brandenburg W, Gilissen L, Kleter G, Wagenaar J (2002) The Biosafety Files, a new link in biosafety information. Biotechnology and Development Monitor 49: 13-14. http://www.biotech-monitor.nl/4905.htm
McLean MA, Frederick RJ, Traynor P, Cohen JI, Komen J (2002) A conceptual framework for implementing biosafety: Linking policy, capacity and regulation. International service for national agricultural research (ISNAR), Briefing paper $\mathrm{n}^{\circ}$ 47. ftp://ftp.cgiar.org/isnar/publicat/bp-47.pdf

OECD (2002) Glossary of statistical terms. Organisation for Economic Co-operation and Development (OECD), Paris, France. http://stats.oecd.org/glossary/detail.asp?ID=5103

One World (2010) Capacity building guide. http://uk.oneworld. net/guides/capacitybuilding\#top

Secretariat of the Convention on Biological Diversity (2000) Cartagena Protocol on Biosafety to the Convention on Biological Diversity, Montreal, Canada. http://www.cbd.int/ biosafety/protocol.shtml

Secretariat of the Convention on Biological Diversity (2004) Action Plan for Building Capacities for the Effective Implementation of the Cartagena Protocol on Biosafety. Decision BS-I/5 Capacity-building, Annex I. First Meeting of the Conference of the Parties to the Convention on Biological Diversity serving as the Meeting of the Parties to the Cartagena Protocol on Biosafety (COP-MOP 1), Kuala Lumpur, Malaysia, 23-27 February 2004. http://www.cbd.int/ decision $/ \mathrm{mop} /$ ?id $=8287$

Secretariat of the Convention on Biological Diversity (2005) Report on the Needs and Priorities of Countries for the Implementation of the Protocol and Possible Measures for Addressing Them, Including Opinions for Enhancing the Effectiveness and Adequacy of Financial Assistance. $\mathrm{UNEP} / \mathrm{CBD} / \mathrm{BS} / \mathrm{COP}-\mathrm{MOP} / 2 / \mathrm{INF} / 7$, Second Meeting of the Conference of the Parties to the Convention on Biological Diversity serving as the Meeting of the Parties to the Cartagena Protocol on Biosafety (COP-MOP 2), 30 May3 June 2005, Montreal, Canada. http://www.cbd.int/doc/ meetings/bs/mop-02/information/mop-02-inf-07-en.pdf

Secretariat of the Convention on Biological Diversity (2009) Report of the sixth meeting of the liaison group on capacitybuilding for biosafety. UNEP/CBD/BS/LG-CB/6/3, 12-13 March 2009, San José, Costa Rica. http://www.cbd.int/doc/ meetings/bs/bslgcb-06/official/bslgcb-06-03-en.pdf

Secretariat of the Convention on Biological Diversity (2010) Report of the seventh meeting of the liaison group on capacity-building for biosafety. UNEP/CBD/BS/LG-CB/7/2, 4-5 February 2010, Siem Reap, Cambodia. http://www.cbd. int/doc/meetings/bs/bslgcb-07/official/bslgcb-07-02-en.pdf

UNEP-GEF Biosafety Unit (2005) Building Biosafety Capacity in Developing Countries. Experiences of the UNEP-GEF Project on Development of National Biosafety Frameworks. Division of the Global Environment Facility (GEF), United Nations Environment Programme (UNEP), Nairobi, Kenya. http://www.unep.org/biosafety/ files/UNEPGEFstudyVersion170605.pdf 